\title{
EFFECTS OF ATORVASTATIN COMBINED WITH AEROBIC RESISTANCE EXERCISE ON LUNG FUNCTION
}

\author{
XIAOLING XIONG ${ }^{1}$, JUN LI ${ }^{2}$ and CHUNYAN LI ${ }^{1 *}$
}

'Hubei Key Laboratory of Sport Training and Monitoring, College of Health Science, Wuhan Sports University, Wuhan, 430079, China

${ }^{2}$ Dept of Obstetrics, Renmin Hospital of Wuhan University, Wuhan, 430060, China

\begin{abstract}
Chronic obstructive pulmonary disease (COPD) is a chronic respiratory disease seriously threatening the quality of life of patients. In the treatment of patients with COPD at a stable stage, aerobic resistance exercise is not only needed to help patients recover their exercise function but also medication is needed to inhibit the production of inflammatory factors. This study discussed the feasibility of combining aerobic resistance exercise with medication to treat COPD. One hundred forty-seven patients with COPD who were hospitalized in the internal medicine department of our hospital from October 2015 to June 2019 were selected, and 120 patients were selected as subjects of this experiment on a voluntary basis. Aerobic resistance exercise therapy, atorvastatin, and combination therapy were performed respectively, and the patients' physical fitness indicators, levels of inflammatory cytokines, and cardiopulmonary function were observed to evaluate treatment plans. After receiving the treatment of aerobic and obstructive exercise combined with atorvastatin, COPD patients showed improvement in the indicators related to physical fitness, including decreased body fat percentage, waist-to-hip ratio, BMI, blood sugar, hbalc content, and blood lipid, decreased levels of inflammatory factors like IL-8, IL-6, TNF- $\alpha$, and some recovery in cardiopulmonary function. A combination of aerobic and obstructive exercise with atorvastatin can better treat COPD and improve the exercise function and cardiopulmonary function of patients. However, the clinical application of this treatment plan needs more cases to verify, and clinical application also needs to adjust the plan appropriately according to individual differences.
\end{abstract}

Keywords: aerobic resistance exercise, atorvastatin, Chronic obstructive pulmonary disease (COPD)

Chronic obstructive pulmonary disease $(\mathrm{COPD})$ is a chronic respiratory disease with the highest prevalence at present. It is mainly characterized by airflow limitation, high morbidity, lingering illness, recurrent attacks, high mortality, inflammatory reaction, heavy social and economic burden, and severe threat to patients' quality of life. Pulmonary function rehabilitation training and atorvastatin can improve the pulmonary function of COPD patients, improve exercise endurance and cardiopulmonary function (1-2). Aerobic exercise is a common pulmonary rehabilitation training method, but studies have shown that skeletal muscle atrophy will occur in patients with COPD, which is also one of the important factors for the decline of patients' quality of life (3). Resistance in routine aerobic training can increase muscle strength, so aerobic resistance to training has a particular significance for the treatment of COPD. Bronchodilators and glucocorticoids are often used in the clinical treatment of COPD. Recent studies have shown that atorvastatin, a commonly used lipid-regulating drug in clinical practice, has anti-inflammatory, antioxidant, and immunomodulatory effects, and can reduce the mortality and hospitalization risk of COPD patients (4-5). However, there are few studies on the treatment of COPD by atorvastatin. The purpose of this study was to investigate the therapeutic efficacy of aerobic resistance exercise combined with atorvastatin in the treatment of COPD and to provide a basis for the treatment of COPD.

\section{EXPERIMENTAL}

\section{Materials \\ Patients}

147 patients with COPD hospitalized in the internal medicine department of our hospital from

\footnotetext{
* Corresponding author: e-mail: lichunyan452@yandex.com
} 
October 2015 to June 2019 were selected as experimental subjects. 120 patients were randomly assigned to the experimental group $(\mathrm{n}=90)$ and the control group $(n=30)$ on a voluntary basis (Table 1), including 65 males and 55 females, with an age of $63.6 \pm 7.6$ years and a course of disease of 8.1 \pm 5.1 years.

\section{Inclusion and exclusion criteria}

According to the Chinese medical association's guidelines for the diagnosis and treatment of COPD (2007 revision) (6). The inclusion criteria were as follows: (1) patients diagnosed with COPD who volunteered for observation; (2) patients who had not taken statins in the 2 weeks before onset and did not have severe liver and kidney dysfunction; (3) serum creatine kinase was higher than five times the standard high value, alanine transaminase was higher than 3 times the normal high value, serum creatinine concentration was greater than $221 \mathrm{~mol} / \mathrm{L}$, and patients with myalgia during the medication needed to stop the drug treatment. The exclusion criteria were as follows: (1) patients with major comorbidities such as liver, renal, heart disease, or diabetes mellitus; (2) patients who have used over 2-week oral or parenteral corticosteroids during the past two years.

\section{Treatment}

According to the pathological causes of patients with COPD and its physical characteristics and willingness, established the exercise prescription of the present study: aerobic and resistance exercises such as taijiquan and jogging. According to the patient's interest and mastery degree determine an aerobic and resistance exercise. According to the formula: when oxygen consumption $\mathrm{X} \%=$ Hrrest+(HRMax-Hrrest $) \times X \%$ oxygen consumption calculation, the experimental group exercise set to moderate heart rate, oxygen consumption 40-60\%, The exercise level of the experimental group was set as moderate-intensity heart rate and $40-60 \%$ oxygen consumption. Exercise prescription is carried out under the guidance of professionals. The standard gradient of resistance training was set as $45 \%, 50 \%$, $55 \%$, and $60 \%$ of the maximum strength. For drug treatment, the patients were treated with $40 \mathrm{mg}$ /day of atorvastatin. The experimental group was further divided into three subgroups. Experiment group 1 received aerobic resistance only; Experiment group 2 received atorvastatin treatment only; Experiment group 3 received both aerobic resistance and atorvastatin treatment. The negative control group exercised based on patients' previous exercise habits and did not receive drug treatment.

Interleukin 6 (IL-6) and interleukin 8 (IL-8), tumor necrosis factor-alpha (TNF alpha) measurement

The patients' fasting venous blood was collected before treatment and 6 months after treatment and was measured by enzyme-linked sandwich immunoassay (ELISA) using a kit purchased from the immunology department of the fourth military medical university. Physical fitness test and measurement of 4 indexes of blood lipid: the body density was calculated by using the formula body fat percentage $(\%)=(4.570 /$ body density -4.142$) \times 100$ calculated densities. Total fat is equal to bodyweight multiplied by body fat percentage. Fat loss weight (lean weight) is weight minus the total fat weight. Blood glucose and glycosylated hemoglobin indicators: fasting blood glucose (FBG) concentration and HBAIC in patients were measured with a blood glucose meter produced by Novo Nordisk and a Boehringer Manheim kit from Germany, respectively. Serum cholesterol (Tch), high-density lipoprotein (HDL), low-density lipoprotein (LDL), and triglyceride (TG) were measured by 721 spectrophotometers.

\section{Lung function measurement}

The ms-diffusion lung function meter produced by Yager company in Germany was used to measure the lung function of the patients. The lung function indicators were forced expiratory volume (FEV1) and forced vital capacity (FVC\%).

Table 1. Condition of experimental subjects.

\begin{tabular}{|c|c|c|c|}
\hline & Number & Age & Year \\
\hline Experimental group 1 (aerobic resistance) & 30 & $65.46 \pm 2.67$ & $8.81 \pm 3.41$ \\
\hline Experimental group 2 (atorvastatin) & 30 & $62.87 \pm 3.26$ & $8.14 \pm 3.18$ \\
\hline $\begin{array}{c}\text { Experimental group 2 (aerobic resistance } \\
+ \text { atorvastatin) }\end{array}$ & 30 & $63.27 \pm 2.86$ & $7.83 \pm 2.16$ \\
\hline Control group & 30 & $62.91 \pm 2.48$ & $7.68 \pm 3.22$ \\
\hline
\end{tabular}



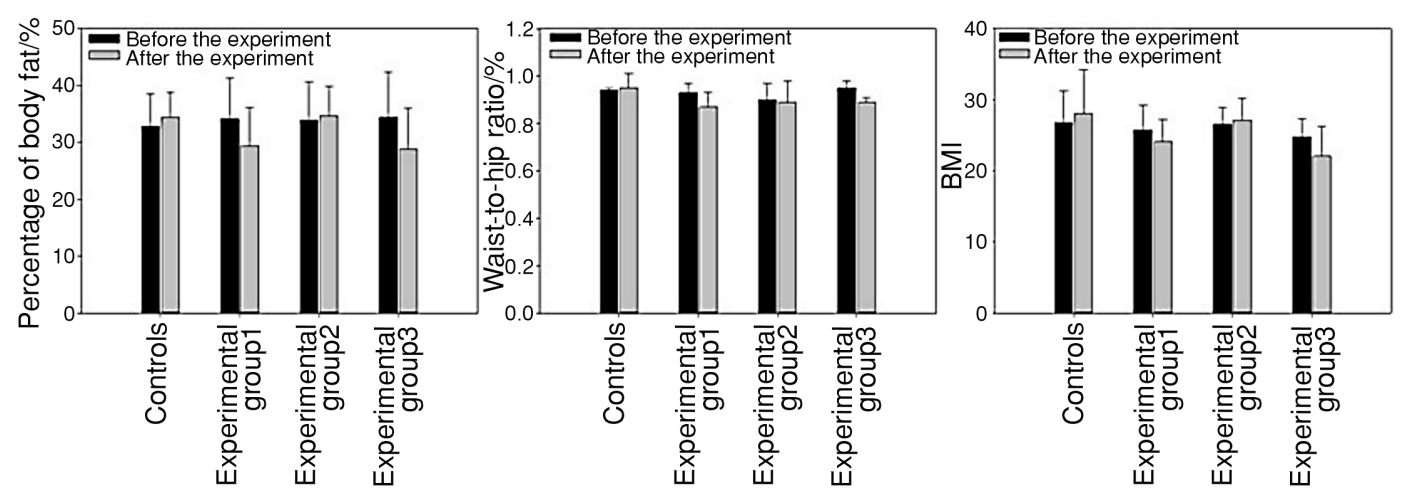

Figure 1. Changes in physical health indicators.

\section{Statistical analysis}

Data were analyzed using the statistical package for social sciences (SPSS) software version 13 (IBM Inc, Chicago, IL, USA). The normality of continuous variables was assessed using the Shapiro-Wilk test. Normally distributed variables were presented as mean \pm standard deviation (SD). The P-value less than 0.05 was considered statistically significant.

\section{RESULTS AND DISCUSSION}

Effects of aerobic resistance exercise and combined treatment with atorvastatin on patients' physical fitness indicators

After systematic moderate-intensity aerobic exercise plus resistance training and atorvastatin treatment, the body indicators changed to different degrees. Body fat percentage, waist-to-hip ratio, and BMI were compared with the pre-experiment data (Figure 1).

After simple exercise treatment, the above indexes were significantly reduced, taking atorvastatin alone had less effect on these indicators. In combination with aerobic plus resistance exercise and atorvastatin, the effect was superior to that of the individual treatment. Before and after the experiment, the above indexes of the control group did not change significantly. It shows that the exercise prescription adopted in this study has a very positive effect on improving the physical condition of patients, and the combination of aerobic resistance exercise and atorvastatin has a better effect.

Effects of aerobic resistance exercise and combined treatment with atorvastatin on blood glucose and hba1c in patients

The blood glucose (FBG) concentration and HBAIC indexes of the group treated with aerobic resistance exercise, aerobic resistance exercise, and atorvastatin were significantly lower than those before the experiment. However, there was no significant change in the control group before and after the experiment (Table 3), indicating that aerobic and obstructive exercise treatment could slow down the blood glucose and HBAIC of COPD patients. Previous studies have shown that aerobic resistance exercise can reduce the blood glucose level of patients (7-12). However, there are few studies on the impact of aerobic resistance exercise on COPD. This study not only discussed the impact of aerobic resistance exercise on COPD but also discussed the effect of atorvastatin and the combination of the two in the treatment of COPD. When atorvastatin was treated alone, the changes in these two indicators were not significant. However, when the patients were treated with aerobic resistance exercise and atorvastatin combined therapy, their blood glucose, and HBAIC contents were significantly lower than before the experiment and lower than the group treated with exercise alone, it could be seen that the combination of the two had a better therapeutic effect on the patients

Effects of aerobic resistance exercise and atorvastatin combined therapy on the serum lipid of patients

Studies have shown that reduced levels of Tch, TG, HDL, and LDL in the blood can effectively relieve and prevent the deposition of cholesterol in the arterial wall, thus reducing the incidence of cerebrovascular diseases (13). In this study, we investigated the effects of different treatment regimens on Tch, TG, HDL, and LDL in patients. The experimental results showed that the indicators of Tch, TG, and LDL in the group treated with aerobic 

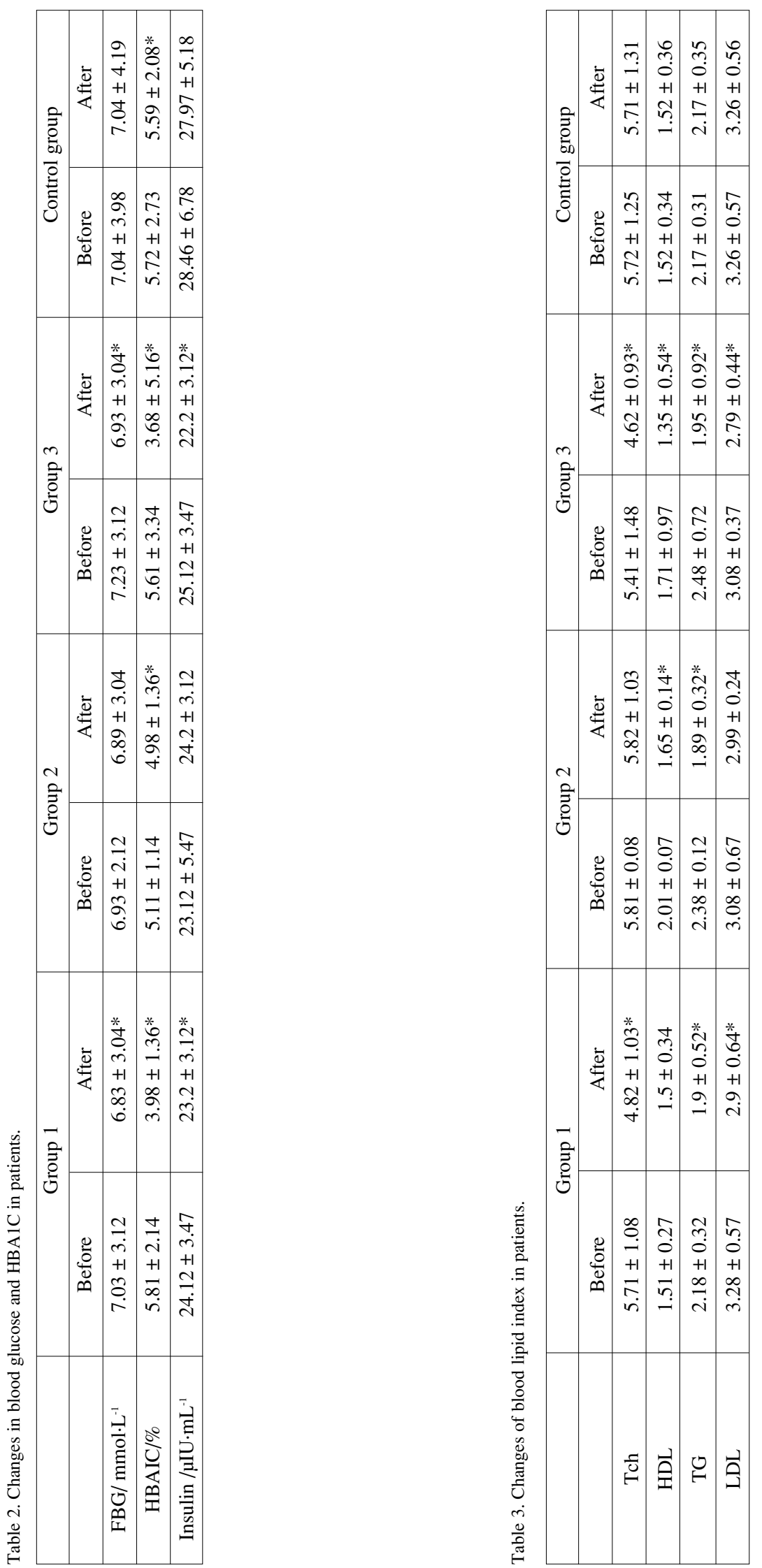
resistance exercise, aerobic resistance exercise, and atorvastatin were significantly lower than those before the experiment. However, there was no significant change before and after the experiment in the control group (Table 4). Therefore, aerobic resistance exercise therapy can slow down the indicators of Tch, TG, and LDL in COPD patients. Atorvastatin regulates lipid, TG, and HDL levels decreased for patients treated with atorvastatin alone. The effect of exercise on these indicators may be that exercise improves the activity of lipoprotein enzymes in muscle and adipose tissues and accelerates the transfer of cholesterol and phospholipid to HDL (14-17). The effect of atorvastatin on these indicators may be other mechanisms. After combining aerobic resistance exercise with atorvastatin in the treatment of COPD patients, these indicators were significantly lower than those of the control group (Table 3), indicating that the combination of the two treatments had a better therapeutic effect on patients.

The effect of aerobic and resistance exercise and atorvastatin combined treatment on patients with IL-8, IL-6, and TNF - $\alpha$

Inflammatory cells activated in OPD patients migrate to lung tissues and release a variety of inflammatory cytokines, including il-8, il-6, TNFlevels, etc. (18-20). Therefore, monitoring the changes in these indicators in patients can better evaluate the treatment regimen. The IL- 8 , IL- 6 and TNF- levels of atorvastatin, aerobic resistance exercise, and atorvastatin combined treatment group after the experiment were significantly lower than those before the experiment. However, there was no significant change before and after the control group
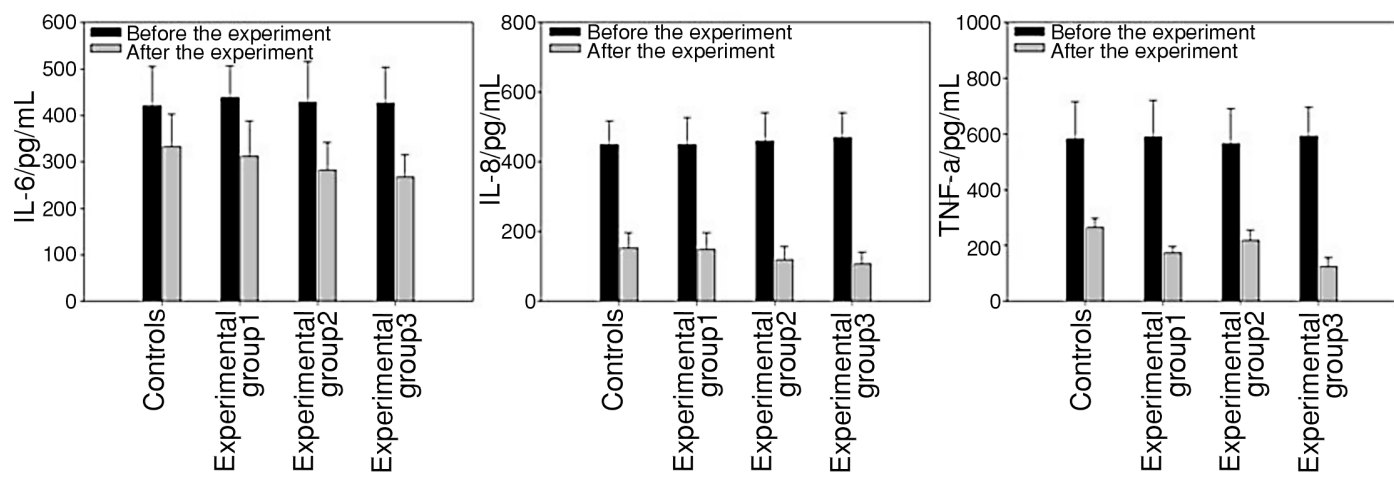

Figure 2. Effect of different treatment on patients with IL -8 , IL 6 and TNF $-\alpha$.
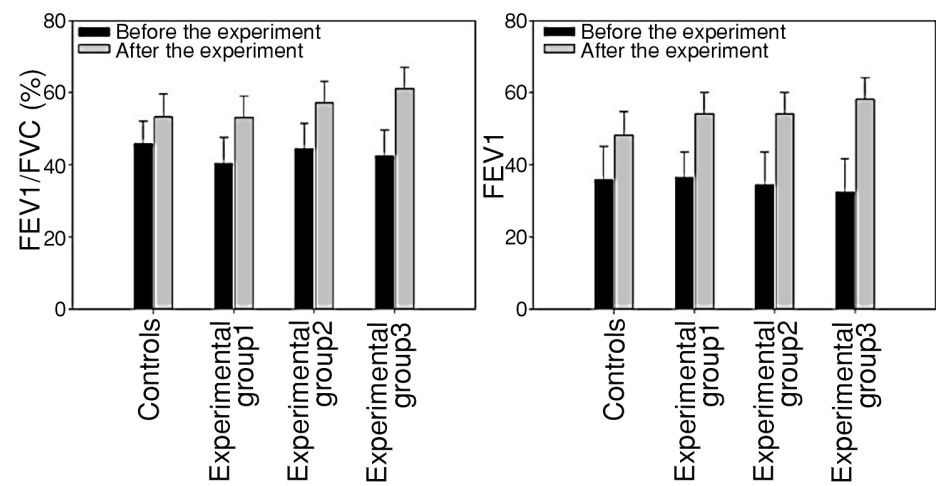

Figure 3. Effects of different treatments on patients with FEV1/FVC and FEV1. 
(Fig. 2), indicating that atorvastatin treatment can slow down the content of inflammatory cytokines in COPD patients. Previous studies have shown that atorvastatin can reduce the level of inflammatory cytokines in patients (21-24). However, there are few studies on the effect of aerobic resistance exercise on inflammatory cytokines. This study not only discussed the effect of aerobic resistance exercise on inflammatory cytokines in COPD patients but also discussed the effect of atorvastatin and the combination of the two in the treatment of COPD. When atorvastatin alone was treated, the changes in inflammatory cytokines were significantly lower than before the experiment. After aerobic exercise treatment, the level of inflammatory cytokines decreased, but there was no significant difference compared with the control group. However, when the levels of inflammatory cytokines in the patients treated with aerobic resistance exercise and atorvastatin were significantly lower than before the experiment and lower than the group treated with exercise alone, it could be seen that the combination of the two treatment was more effective for the patients.

Effects of aerobic resistance exercise combined with atorvastatin on the cardiopulmonary function of patients

Control group patients with FEV1 / FVC, FEV1 indicators have no significant changes before and after the experiment, but after aerobic and resistance exercise therapy, atorvastatin treatment and combination of the above index significantly increased after the treatment (Fig. 3), indicating aerobic and resistance exercise therapy, atorvastatin treatment all can improve the patients' cardiopulmonary function, combination therapy on the patients has a better effect

COPD is a kind of lung disease with chronic inflammation in the airway and lungs. In daily life, the inflammatory cells activated by smoke and bacteria will migrate to the lung tissue and release a variety of inflammatory cytokines such as IL-8, IL- 6 and TNF- $\alpha$. These cytokines exhibited unique roles in inflammatory diseases and could be targeted for control of certain diseases. In addition, these cytokines were factors related to COPD in patients $(32,33)$. COPD often leads to varying degrees of cardiac function damage, resulting in decreased motor function and skeletal muscle atrophy, which ultimately affects the quality of life of patients (1-3, 5-7). Therefore, the treatment of patients with COPD at a stable period requires not only appropriate exercise to help patients recover their exercise function, but also drug therapy to inhibit the production of inflammatory factors. This study discussed the feasibility of combining aerobic exercise with drugs to treat COPD. Studies have shown that lung rehabilitation training such as aerobic exercise and abdominal breathing can stimulate the diaphragm and internal and external abdominal wall muscles and other respiratory muscles, increase the ability of lung ventilation, such this kind of training can improve lung ventilation to a certain extent, reduce hypercapnia. Resistance exercises can increase muscle strength, improve muscle function, and help patients improve motor function (11-14, 21-23). Studies have also shown that prolonged aerobic resistance training can help diabetic patients to control blood glucose and improve their muscle strength and cardiovascular function $(13,16,20)$. At the same time, a study was conducted on patients with coronary heart disease, and it was found that aerobic combined elastic band impedance exercise could improve oxygen uptake and respiratory function of patients (22). In the study, the exercise treatment program we provided was the aerobic resistance exercise program. By using this treatment program, COPD patients improved their physical fitness and cardiopulmonary function. This indicates that aerobic resistance exercise can effectively treat COPD patients.

\section{CONCLUSION}

Atorvastatin is a class of reductase inhibitors, and the full name is 3-hydroxy3-methyl-glutaryl coenzyme A (HMG - COA), commonly used in clinical lipid regulation and the prevention of cardiovascular disease. Atorvastatin also has antiinflammatory, antioxidant, and immune regulatory effects. COPD patients under the stimulus of smoke activated inflammatory cells migrate to the lung tissue and release a variety of inflammatory cytokines, such as IL 8, IL - 6, TNF alpha. Excessive amounts of inflammatory cytokines will lead to the accumulation of inflammatory cells in lung tissue, on the other hand, the activity of inflammatory cells will be improved and more inflammatory cytokines will be secreted to gather around inflammatory cells, forming a vicious circle between inflammatory cells and inflammatory cytokines and jointly causing deep lung tissue damage (20-21, 25-31). Monitoring changes in IL-8, IL-6, and TNF- in patients can better evaluate the treatment regimen. After atorvastatin treatment, the levels of inflammatory cytokines in COPD patients were significantly lower than 
before the experiment. The level of inflammatory cytokines could also be reduced by aerobic exercise therapy, but the degree was significantly lower than that of atorvastatin. When combined with aerobic resistance exercise and atorvastatin, the level of inflammatory cytokines in patients was significantly lower than that before the experiment, and the effect was also better than that of the group treated with exercise alone or atorvastatin alone, indicating that the combination of the two treatment had a better therapeutic effect on patients. In summary, the combination of aerobic resistance exercise and atorvastatin can effectively improve the cardiorespiratory function and exercise endurance of COPD patients, and reduce inflammatory factors. However, the clinical application of this treatment plan needs to be verified by more cases, and it also needs to be appropriately adjusted according to individual differences.

\section{Acknowledgment}

This work was supported by "physical education and health promotion" of the dominant academic group in Hubei province (81971661, B2017232). Thanks for the support of "physical education and health promotion" of the dominant academic group in Hubei province.

\section{Conflicts of interest}

The authors declare no conflict of interest.

\section{REFERENCES}

1. Cai J.G., Chen J.G., He H.: Cell Biol. Toxicol. 25, 435 (2009).

2. Kikugawa K., Machida Y., Kida M., et al.: Chem. Pharm. Bull. 29, 3003 (1981).

3. Wolf f A.A., Hines D.K., Karliner T.S.: Am. J. Physiol. 257, H1032 (1989).

4. Hwang D.F., Wang L.C.: Toxicology 167, 173 (2001).

5. Nieminen M.L., Tuomist O.L., Solatunturi E., Eriksson L., Paasonen M.K.: Life Sci. 42, 2137 (1988).

6. Zhong N., Wang C., Yao W., Chen P., Kang J., et al.: Am. J. Respir. Critical Care Med. 176, 753 (2007).

7. El Idrissi A., Messing J., Scalia J., Trenkner E.: Adv. Exp. Med. Biol. 526, 515 (2003).

8. El Idrissi A., Trenkner E.: Adv. Exp. Med. Biol. 526, 527 (2003).
9. Camerino D.C., Tricarico D., Pierno S., Desaphy J.F., Liantonio A., et al.: Neurochem. Res. 29, 135 (2004).

10. Urso M.L., Clarkson P.M.: Toxicology 189, 41 (2003).

11. Yin D.Z., Chen K.J.: Exp. Gerontol. 40, 455 (2005).

12. Dawson R.Jr., Biasetti M., Messina S., Dominy J.: Amino Acids 22, 309 (2002).

13. Nandhini A.T., Thirunavukkarasu V., Ravichandran M.K., Anuradha C.V.: Singapore Med. J. 46, 82 (2005).

14. Schreuder T.H.A., Munckhof I.V.D., Poelkens F., Hopman M.T.E., Thijssen D.H.J.: Eur. J. Appl. Physiol. 115, 317 (2015).

15. Mohammed J., Derom E., Van Oosterwijck J., Da Silva H., Patrick C.: Physiotherapy 104, 36 (2018).

16. Sherpa C.T., LeClerq S.L., Singh S., Naithani N., Pangeni R., et al.: Chronic Obstr. Pulm. Dis. 2, 281 (2015).

17. Martin S., Wolf Eichbaum D., Duinkerken G., Scherbaum W.A., Kolb H., et a1.: N. Engl. J. Med. 315, 1036 (2001).

18. Alidjinou E.K., Sané F., Engelmann I., Geenen V., Hober D.: Dicov. Med. 18, 273 (2011).

19. Schneider D.A., Von Herrath M.G.: Diabetologia 57, 2009 (2014).

20. Ramakrishna V., Jailkhani R.: Diagn. Pathol. 2, 22 (2007).

21. Hieronymus L., Geil P.: Diabetes Self Manag. 31, 22 (2014).

22. Chang A.M., Halter J.B.: Am. J. Physiol. Endocrinol. Metab. 284, E7 (2003).

23. Dillon N., Chung S., Kelly J., O'Malley K.: Clin. Pharmacol. Ther. 27, 769 (1980).

24. Saitoh S., Shimoda T., Hamamoto Y., Nakaya Y., Nakajima S.: Indian J. Endocrinol. Meatab. 19, 66 (2015).

25. Hahn V., Halle M., Schmidt-Trucksäss A., Rathmann W., Meisinger C., et al.: Diabetes Care 32, 511 (2009).

26. Hui S.S., Hui G.P., Xie Y.J.: PLos One. 9, 1371 (2014).

27. Paffenbarger R.S. Jr, Lee I.M., Kamperl J.B.: World Rev. Nutr. Diet. 82, 210 (1997).

28. Wakabayashi I.: Metab. Syndr. Relat. Disord. 12, 70 (2014).

29. Wei X., E M., Yu S.: Diabetes Res. Clin. Pract. 107, 9 (2015).

30. Choi S.E., Choi K.M., Yoon I.H., Shin J.Y., Kim J.S., et al.: Transpl. Immunol. 13, 43 (2004). 
31. Ezzidi I., Mtiraoui N., Chaieb M., Kacem M., Mahjoub T., et al.: Diabetes Metab. 35, 214 (2009).

32. Chaouat A., Savale L., Chouaid C., Tu L., Sztrymf B., et al.: Chest. 136, 678 (2009).

(C) 2020 by Polish Pharmaceutical Society. This is an access article under the CC BY NC license (http://creativecommons.org/licenses/by-nc/4.0/).
33. Tanaka T., Narazaki M., Kishimoto T.: Cold Spring Harb. Perspect. Biol. 6, a016295 (2014). 